A Barkat, L Benmiloud, M Kabiri. Faculté de Médecine et de Pharmacie de Rabat, Université Souissi, Rabat, Morocco

The objective of our study is to assess the prevalence of infectious perinatal risk situations in the delivery maternity room in Souissi hospital in Rabat, Morocco.

Materials and Methods A prospective study about all infants born in maternity Souissi (Rabat) during one year: from first January to 31 December 2010. We included all newborns symptomatic or not at birth and whose mothers have at least one infectious case histories from the following: rupture of membrane $\geq 12$ hours, chorioamnionitis, urinary tract infection and/or genital, fever $\geq 38^{\circ} \mathrm{C}$ before or in early labor.

Results On a total of 14792 live births, 1602 newborns were prone to a risky infection (10.83\%). Prolonged rupture of membranes showed 1341 cases including 59 newborns were premature. The duration of the rupture was between 12 and 24 hours in $44.30 \%$ of cases, between 24 and 48 hours in $44,37 \%$ of cases and more than 48 hours in $11.33 \%$ of cases. On 133 cases of chorioamnionitis (10.67\%) 129 newborns were full-term and 7 were premature.

The obstetric decision was caesarean section delivery in $25.78 \%$ of cases. $4.74 \%$ of newborns had respiratory distress with a single case of death in the first hours of life. $4.50 \%$ were preterm and $8.86 \%$ were hypotrophy. Hospitalization was required from the outset in 1059 cases among which 133 were hospitalized. For other patients an inflammatory report was requested. The were followed as outpatients.

\section{NUTRI-MEDICINAL PLANTS FOR MANANGEMENT OF CHILDREN DISEASES IN UGANDA: CASE STUDY OF NAMUNGALWE SUB COUNTY, IGANGA DISTRICT}

doi:10.1136/archdischild-2012-302724.0900

'M Kamatenesi-Mugisha, ${ }^{2 P}$ Nalumansi, ${ }^{2} \mathrm{~J}$ Tabuti. 'Biological Sciences, School of Biosciences, Makerere University; ${ }^{2}$ Institute of Environment and Natural Resources, Makerere University, Kampala, Uganda

Nutrition deficiency is a key determinant of an immune deficient body state. There is scientific evidence that plant species effective in disease management confers both nutritional and medicinal benefits to the people using them. The main objective of this study was to identify the plants known to be used in children diseases management and determine their macronutrient and micronutrient composition. To achieve the first objective, a survey was conducted between July 2009 and February 2010 using semi-structured interviews and questionnaires; focused group discussions, participant observation and field visits. Nutrition composition of plants used was determined using standard laboratory methods in the nutrition laboratory in the department of Food Science and Technology, Makerere University. A total of 67 species were documented as plants used in the disease management among children. These species belonged to 38 families and 35 genera. Faboideae (5), Asteraceae (4) and Mimosaceae (4) families had the most number of plant species. Herbs (37.7\%) were the most used plant life forms followed by $(34.4 \%)$ in disease management among the children. Leaves $(58.1 \%)$ were the most used plant parts. These plant species are mainly cultivated (42.6\%). The plants were mainly boiling. Most plant species were used in management of malaria, anemia and diarrhoea among children. The six selected plants, Acacia seyal, Albizia coriaria, Dicliptera laxata, Kalonchoe densiflora, Persea americana and Vernonia amygdalina that were analysed in this study all had macronutrients and micronutrients except phosphorus and sodium. There is a great potential of nutrition supplements using plants in children diseases management.

\section{IS THERE ANY DIFFERENCE BETWEEN THE SYMPTOMATOLOGY AND CLINICAL FINDINGS OF VIRAL AGENTS THAT CAUSED DEHYDRATION?}

\author{
doi:10.1136/archdischild-2012-302724.0901
}

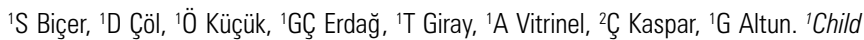
Health and Diseases; ${ }^{2}$ Medical Statistics, Yeditepe Univ Medical Faculty, Istanbul, Turkey

Background and Aims Acute gastroenteritis is responsible for dehydration in many children. The viruses are considered the main agents of gastroenteritis, and these are included by rotavirus, norovirus, adenovirus. Evaluation of the symptoms, clinical findings and hospitalization requirements were aimed in cases of dehydration.

Methods The distribution of age, symptoms, clinical and laboratory findings and hospitalization requirements of viral gastroenteritis cases who have moderate to severe dehydration were evaluated retrospectively. A total of 156 patients with moderate to severe dehydration caused by acute viral gastroenteritis were evaluated. Patients were between 3 months to 16 years of age (mean: 38.7 months). Rotavirus, Norovirus and Adenovirus were detected by immunochromatographic method, as the causes of gastroenteritis.

Results Dehydration were detected in 156 patients with acute gastroenteritis (156/278), which included patients with Rotavirus (48\%), Norovirus (41\%) and Adenovirus (13.5\%), respectively. Norovirus was mostly detected (51.8\%) in the first 24 months of age, however, Rotavirus was mostly detected in $>24$ months of age $(61.3 \%)$. The common symptoms of all patients were vomiting, diarrhea, abdominal pain and malaise, although fever was seen mostly the cases with Rotavirus. A total of 59 patients were hospitalized, they were Rotavirus cases mostly ( $\mathrm{n}=35,59.3 \%$ ).

Conclusions The main agents of acute gastroenteritis which caused in dehydration were Norovirus and Rotavirus in our patients. Norovirus was the mostly detected agent in infants and young children who were $<24$ months of age. Rotavirus was detected in the most of hospitalized patients, it had caused to most of the severe symptoms.

\section{ANTIMICROBIAL UTILIZATION PATTERN IN RESPIRATORY TRACT INFECTIONS AMONG PEDIATRIC POPULATION IN AJMAN, UAE}

doi:10.1136/archdischild-2012-302724.0902

${ }^{1} \mathrm{M}$ Hassan, $2 \mathrm{LJ}$ John, ${ }^{3} \mathrm{M}$ Hassan. 'Pharmaceutics; '2Pharmacology, Gulf Medical University; ${ }^{3}$ Pediatrics, Gulf Medical College Hospital, Ajman, United Arab Emirates

Background and Aims Respiratory tract infection (RTI) among children is a leading cause of sickness among school children and parental absenteeism from work. It is associated with hospitalization and significant morbidity. Antimicrobials play an integral role in management of RTIs but irrational use is too common. Hence, this study aimed to determine the prescribing patterns of antimicrobials among children attending the outpatient department (OPD) of pediatrics in GMC Hospital, Ajman.

Methods A cross-sectional drug utilization study was conducted using the prescriptions from the medical records of patients (aged 0-12 years) diagnosed with RTIs during January 2011 at GMC Hospital, Ajman. The demographic data, clinical diagnosis and antibiotic prescription were analyzed using descriptive statistics (SPSS 19).

Results A total of 488 patients (20.2\%) presented with RTI to the OPD of pediatrics. Male to female ratio was 1.24. Majority of the patients were Egyptians followed by Emiratis. Majority of children $225(46 \%)$ were between 1-5 years of age. Combination of upper and lower respiratory tract infections (URTI \& LRTI) accounted for 187(38\%), URTI 208(23\%), and LRTI 93(19\%). The most frequently prescribed drug categories for treatment of RTIs was antimicrobial 